\title{
Rapidly growing pediatric trampoline-related injuries in Korea: a 10-year single center re- trospective study
}

\author{
Min Jeng Cho, MD, Jihoon Kim, MD, Sung Jeep Kim, MD, Kyu Hyouck Kyoung, MD, Min Ae Keum, MD, Sung Kyun Park, MD \\ Department of Surgery, Ulsan University Hospital, University of Ulsan College of Medicine, Ulsan, Korea
}

Purpose: Several published policy statements have warned against the risks associated with trampoline use and recommended safety guidelines. However, few studies have focused on trampoline-related injuries in Korea. This study aimed to assess the incidence and characteristics of pediatric trampolinerelated injuries presented to Ulsan University Hospital.

Methods: We retrospectively reviewed the medical records of children aged $<16$ years with trampolinerelated injuries who visited our Emergency Department between 2008 and 2017.

Results: Over the 10-year period, 178 trampoline-related injuries were reported, which represented a significant increase $(P=0.016)$. Most $(87.6 \%)$ of the injuries occurred during the last 5 study years, and a rapid increase in injuries was observed in children aged $<6$ years. Lower extremity injuries (62.4\%) were the most common, followed by injuries of the upper extremities, head and face, and trunk, including injuries to the neck and spine. Sixty-seven children (37.6\%) had fractures, and proximal tibia fractures were the most common. Fractures were significantly more common in younger children ( $<6$ years old) than in older children $(P=0.026)$.

Conclusion: In Korea, the mechanism of trampoline injury is similar to that of injuries incurred in indoor trampoline parks but is characterized by smaller spaces and multiple users. Trampoline use and the incidence of trampoline-related injuries in children aged $<6$ years are increasing rapidly. Prohibiting the use of trampolines for children aged $<6$ years, restricting simultaneous use by multiple children, and ensuring adult supervision should be strictly emphasized. Public awareness and policy guidelines are needed to reduce the incidence of trampoline-related injuries.

Key words: Trampoline, Injuries, Pediatric, Fracture, Korea

\section{Introduction}

Trampolines are a popular activity among children. Several institutions have reported a significant increase in trampoline-related injuries in recent decades. ${ }^{1-4)}$ The evolving patterns of trampoline use and injury resulted in a series of published policy statements from the American Academy of Pediatrics (AAP) in 1977, 1981, 1999, and 2012..$^{5-8)}$ These statements all discouraged recreational and playground use of trampolines and urged caution with and further study of trampoline use in supervised training and physical education settings. Nevertheless, estimates from the National Electronic Injury Surveillance System in the United States show that trampoline injuries result in nearly 100,000 Emergency Department (ED) visits each year. ${ }^{5,9)}$ In addition to fractures of the extremities, serious injuries have been reported, including cervical spine and skull fractures. ${ }^{10,11)}$

Trampoline activity in Korea has unique environmental differences. In Western countries,

Copyright (c) 2019 by The Korean Pediatric Society

This is an open-access article distributed under the terms of the Creative Commons Attribution NonCommercial License (http://creativecommons.org/ licenses/by-nc/4.0/) which permits unrestricted noncommercial use, distribution, and reproduction in any medium, provided the original work is properly cited. 
backyard trampolines are common, and large-sized indoor trampoline parks have also emerged recently. In Korea, trampolines are being installed in small indoor playgrounds called "kids' cafes" and small playrooms in restaurants. Due to space limitations, backyard trampolines are not popular in Korea. The AAP has warned against the use of trampolines for children under 6 years old, ${ }^{5)}$ and recommend that safety measures for trampoline use should include constant adult supervision, adequate protective padding, 1 jumper per trampoline, and avoidance of flips and somersaults. Guidelines for these safety measures are difficult to apply in Korea, and few individuals are aware of them.

Recently, the number of ED patients has increased concomitant with the rate of trampoline installations in restaurants and "kids' cafes." However, few published studies have focused on trampolinerelated injuries in Korea. ${ }^{12,13)}$ The aim of this study was to assess the incidence and characteristics of pediatric trampoline-related injuries in Ulsan University Hospital.

\section{Materials and methods}

Between 2008 and 2017, all children < 16 years old with trampoline-related injuries who visited the ED of Ulsan University Hospital were included in the study. Ulsan is a city with a population of 1.17 million, and Ulsan University Hospital is the largest hospital and the only level 1 trauma center, with approximately 2,500 pediatric trauma ED visits per annum. All patient records (type of injury, localization, and treatment) and all radiographs were reviewed. Demographic data were included, such as age, sex, and month of the accident. Approval was granted by the Ulsan University Hospital Institutional Review Board (IRB) (2018-08-004). Informed consent was exempt from the IRB.

Several injury types were grouped together for analysis into the following categories: (1) fracture (fracture and nerve damage with

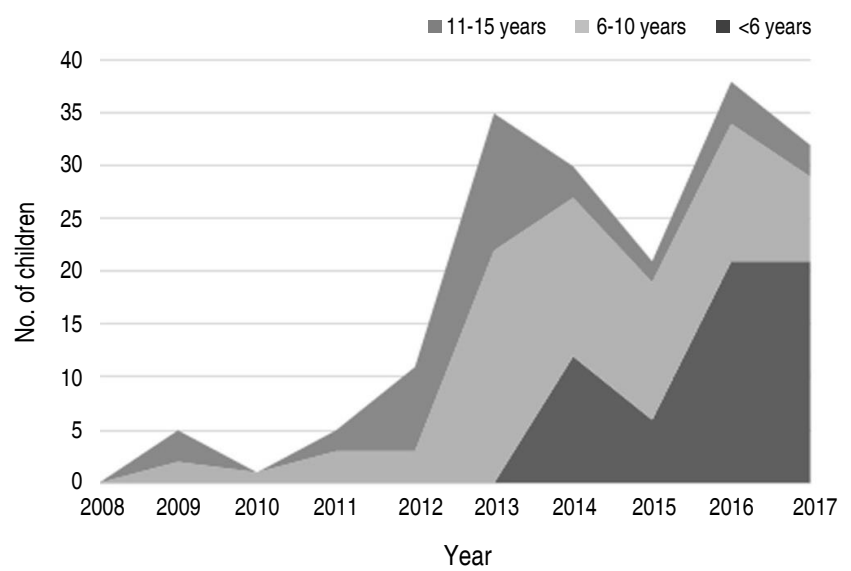

Fig. 1. Number of visits to the Emergency Department due to trampolinerelated injuries by year. fracture); (2) sprain and dislocation (sprain or strain); (3) concussion (concussion, internal organ injury to head, and headache); (4) laceration (laceration, puncture wound, and skin avulsion); (5) contusion or abrasion (contusion or abrasion and superficial hematoma); and (6) other (all remaining injuries not previously specified). The patients were grouped into 3 age categories (0 to 5, 6 to 10, and 11 to 15 years). The cutoff age of 6 years was based on the AAP's recommendation against the use of trampolines for children aged $<6$ years. The injured body regions were categorized as follows: (1) upper extremity (shoulder, upper arm, elbow, lower arm, wrist, hand, and finger); (2) lower extremity (upper leg, knee, lower leg, ankle, foot, and toe); (3) head and face (face, eye area, nose, eyeball, mouth, lips, tongue, teeth, and ear); and (4) trunk, including the spine and neck.

The data were analyzed using IBM SPSS Statistics ver. 22.0 (IBM Co., Armonk, NY, USA). Demographics, injury features, and hospital admission were compared using $t$ tests and the chi-square test. We used chi-square test for trend analysis to identify annual variations. $P$ values less than 0.05 indicated significant differences.

\section{Results}

\section{ED visit trends}

A total of 178 children with trampoline-related injuries were identified. The male:female ratio was 105:73, and the mean age of the patients was $7.1 \pm 3.3$ years. Fig. 1 shows that the trampolinerelated injuries significantly increased over the 10 years investigated $(P=0.016)$, and the majority (87.6\%) of the injuries occurred during the last 5 years of the study. A significant increase was observed in patients under 6 years old when the patients were divided into 3 age groups $(P=0.015)$. Injuries occurred at similar frequencies throughout the month and did not show any seasonal trends (Fig. 2).

\section{Fracture site, injury type, and mechanism}

Fig. 3 shows the fracture sites and injury types. The lower ex-

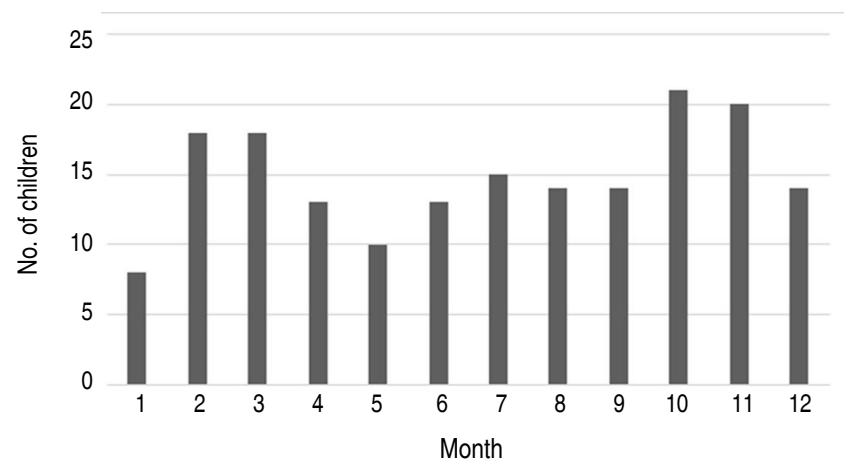

Fig. 2. Number of visits to the Emergency Department due to trampolinerelated injuries between 2008 and 2017 by month. 
tremities were the most frequent site of injuries ( $n=111,62.4 \%)$, and 41 of these injuries involved fractures. Thirty-four patients (19.1\%) had upper extremity injuries, of which 22 involved fractures. The total number of injuries was more frequent in the lower extremities, but the ratio of fractures in the injured patients was higher in the upper extremities $(P=0.007)$. Overall, the most common site of fracture is the proximal metaphysis of the tibia $(n=19)$. Two patients had a skull fracture, one of which was accompanied by an epidural hematoma. A lumbar compression fracture occurred in a 10-yearold patient who bumped his back against another person's knee. He had no neurological symptoms and recovered after conservative treatment. One patient had a laceration of urethral meatus.

Fig. 4 shows the injury mechanisms. Twisted ankle or knee was the most common (28.7\% of injuries). The incidence of fractures was very high in cases involving landing wrong, fell off trampoline, or injury involved another jumper, but no statistical significance $(P=$ 0.071).

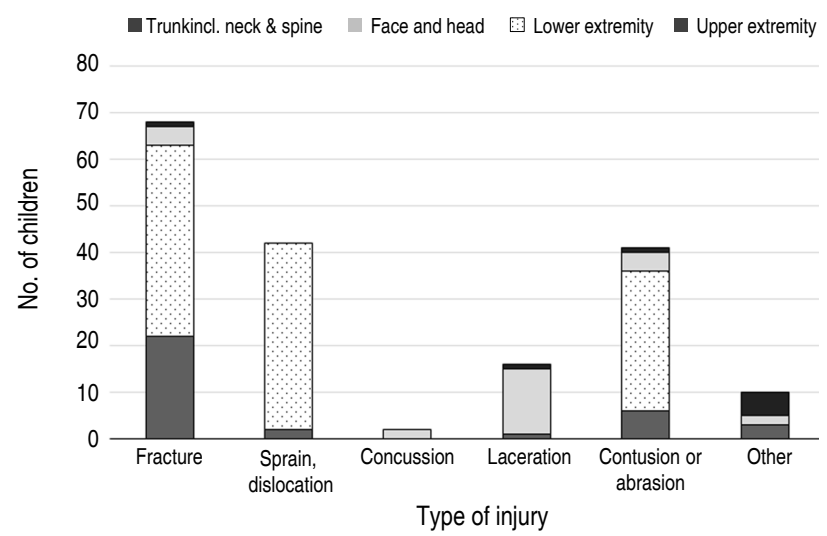

Fig. 3. Types and locations of the trampoline-related injuries.

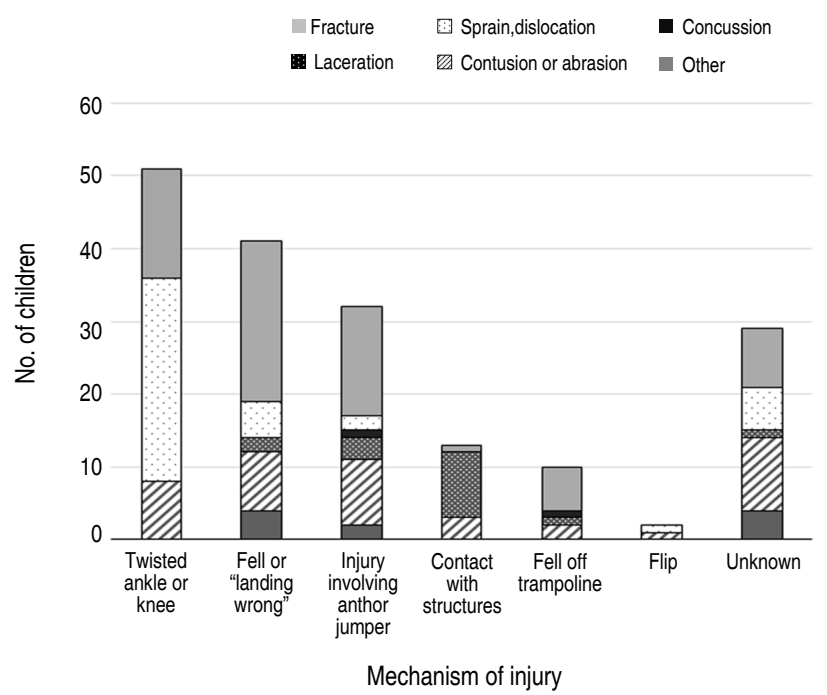

Fig. 4. Types of trampoline-related injuries by mechanism.

\section{Patterns of injury by age}

Table 1 shows the fracture sites, injury types and mechanisms according to the age groups. Children aged 0 to 5 years accounted for 33.7\%, children aged 6 to 10 years accounted for 44.9\%, and children aged 11 to 15 years accounted for $21.3 \%$ of the total cases. Injuries of the lower extremities occurred more frequently in younger children, whereas injuries of the head, face and trunk were more frequent in older children. However, none of these differences were significant. Fractures were significantly more common in younger children $(P=0.026)$. In children $<6$ years of age, fractures were high as $50 \%$ of the total number of injuries.

Twelve patients (6.7\%) underwent surgery, and the remaining patients were treated with splints and conservative therapy. No complications occurred during follow-up.

\section{Discussion}

This study shows that the trampoline-related injuries significantly increased over the 10 years, especially in children $<6$ years old.

Table 1. Trampoline-related injuries treated in one emergency department by age

\begin{tabular}{lcccc}
\hline Variable & $<6$ Years & $6-10$ Years & $11-15$ Years $P$ value \\
\hline No. of cases & $60(100)$ & $80(100)$ & $38(100)$ & \\
Body region injured & & & & 0.199 \\
Upper extremity & $11(18.3)$ & $17(21.3)$ & $6(15.8)$ & \\
Lower extremity & $42(70)$ & $49(61.3)$ & $20(52.6)$ & \\
Face and head & $7(11.7)$ & $10(12.5)$ & $9(23.7)$ & \\
Trunk including neck and spine & $1(1.7)$ & $4(5)$ & $3(7.9)$ & \\
Type of injury & & & & 0.006 \\
Fracture & $30(50)^{\star}$ & $28(35)^{\dagger}$ & $9(23.7)^{\ddagger}$ & 0.026 \\
Sprain and dislocation & $6(10)$ & $22(27.5)$ & $14(36.8)$ & \\
Concussion & $2(3.3)$ & $0(0)$ & $0(0)$ & \\
Laceration & $5(8.3)$ & $5(6.25)$ & $6(15.8)$ & \\
Contusion or abrasion & $17(28.3)$ & $17(21.25)$ & $7(18.4)$ & \\
Other & $0(0)$ & $8(10)$ & $2(5.3)$ & \\
Mechanism of injury & & & & 0.146 \\
Twisted ankle or knee & $12(20.0)$ & $26(32.5)$ & $13(34.2)$ & \\
Fell or landing wrong & $18(30)$ & $17(21.3)$ & $6(15.8)$ & \\
Injury involving another jumper & $9(15)$ & $13(16.3)$ & $10(26.3)$ & \\
Contact with structures & $3(5.0)$ & $4(5.0)$ & $6(15.8)$ & \\
Fell off trampoline & $5(8.3)$ & $5(6.3)$ & $0(0)$ \\
Flip & $1(1.7)$ & $1(1.3)$ & $0(0)$ \\
Unknown & $12(20.0)$ & $14(17.5)$ & $3(7.9)$ & \\
\hline & & &
\end{tabular}

Values are presented as number (\%).

*Upper extremity $(n=10)$, lower extremity $(n=20)$, face and head $(n=1)$; One patient had both upper and lower extremity fracture. ${ }^{\dagger}$ Upper extremity ( $\left.n=9\right)$, lower extremity $(n=18)$, face and head $(n=1)$. . Upper extremity $(n=3)$, lower extremity ( $n=3)$, face and head $(n=2)$, trunk including neck and spine $(n=1)$. 
Fractures were significantly more common in children $<6$ years old, and lower extremity injuries and fractures were the most common. Many previous studies have noted that falling from a trampoline, the exposed frame and springs, and the presence of multiple simultaneous users are risk factors for trampoline-related injuries. ${ }^{6-8,14,15)}$ Since the 1999 AAP policy statement, netted trampolines and extending padding to the frame and springs have reduced fall and frame injuries in home trampoline settings. ${ }^{2,5}$ The large indoor trampoline park, which has increased in many countries in recent years, consist of wall-to-wall multiple interconnected trampolines with a padded floor. The observed decrease in falls from trampolines was accompanied by a worrying increasing trend in injuries associated with multiple users. ${ }^{16,17)}$

Since backyard trampolines are rare in Korea, the mechanisms of injury are essentially the same as those for indoor trampoline parks. However, most of the recent increase in trampoline facilities in Korea was due to installations as part of playgrounds in a restaurant or "kids' cafés." Safety rules suggesting that only 1 person per mat are difficult to enforce because the space is occupied by many children on 1 or 2 mats instead of on multiple interconnected trampolines. The AAP recommends limiting the use of trampolines for children under 6 years old, but most trampolines in "kids' cafés" in Korea can be used by children under 6 years old. According to the Korea Consumer Agency, 83\% of "kids' cafés" and 50\% of indoor playrooms in restaurants are equipped with trampolines. ${ }^{18)}$ The exact number of trampoline installations in Ulsan could not be confirmed, but the number of "kid's cafés" was three in 2012 and increased to 28 in 2017. ${ }^{19)}$ In this study, the rapid increase in trampoline-related injuries over the past 5 years seems to be the result of the recent increase in the number of "kid's cafés." In most studies, the peak seasons for trampoline-related injuries are spring and autumn, when children can play in the backyard. ${ }^{20)}$ In contrast to other studies, trampolines in Korea are located in indoor facilities, and thus this study shows the incidence regardless of the season.

The injured body regions and injury type patterns in this study were similar to those reported in previous studies. ${ }^{1,16,17)}$ Due to the wall-to-wall trampoline design, few falls from trampolines occur for indoor trampolines, which may account for the decreased proportions of head and arm injuries. The lower extremities were the most frequent injury sites, and the most common fractures involved the proximal metaphysis of the tibia. ${ }^{21,22)}$ Several studies showed that the presence of multiple children on a trampoline might increase the axial forces to the knee joint and cause the typical proximal tibia fracture known as "trampoline fracture" in children $<6$ years old. ${ }^{23-25)}$ Our report also showed that 15 of 19 patients with fractures involving the proximal metaphysis of the tibia were under 6 years old. This fracture type may appear very subtly on initial radiographs and thus be overlooked. Klimek et al. ${ }^{24)}$ recommend follow-up radiographs after 7-10 days for cases of inconclusive radiographs and persistent pain of the tibia. Hauth et al. ${ }^{26)}$ recommended gene- rous use of MRI in children with pain after initial negative plain radiography. In the present study, the first X-ray was unable to reveal a fracture in a 3-year-old child. Three days later, the pain persisted, and a computed tomography scan revealed a fracture.

Even if no fracture is present, complications of chronic repeated changes to the lower extremities due to jumping can occur. ${ }^{24)}$ Axial forces to the ankle and knee joints may cause growth arrest lines, widening of the growth plates or sclerosis of the metaphysis. Klimek et al. ${ }^{24)}$ reported early closure of the growth plates in followup radiographs in 2 children. In this study, we could not confirm early closure of the growth plates, because no serial X-ray follow up was performed. However, all patients with injuries of the lower extremities (62.4\%) have the potential to develop complications, including early closure of the growth plates. This possibility is especially worrisome in young children. The epidemic increase in young children $<6$ years old over the past 5 years in this study highlights the urgency of addressing the prevention of trampoline-related injuries.

Fractures of the extremities are the most common injuries, but trampoline-related injuries can also be diverse and severe. This study showed various injuries, such as spinal compression fractures, skull fracture, epidural hematoma, and urethral meatus laceration. Casserly et al. ${ }^{277}$ reported vertebral artery dissection that caused a stroke in a 4-year-old boy. Approximately 10\% of trampolinerelated injuries in children result in spinal injuries, primarily of the cervical spine. ${ }^{10,11,28)}$ Wall-to-wall indoor trampolines are more likely to cause a spine injury than a home trampoline without a net due to the assumption that performing more flips is safer with this set-up. Cervical spine injuries occur in somersault maneuvers to a greater extent in older children and adults than in younger children. Arora et al. ${ }^{29)}$ reported that $40 \%$ of spine injuries occurred in adults. In this study, patients with flip-related injuries accounted for 2 cases with only minor injuries. This small number of cases is due to the fact that most trampolines in Korea are not wide enough for users to perform flips, and younger children that cannot flip use trampolines more frequently than older children that can flip.

One of the most important factors in reducing the risk of trampoline injuries is adult supervision. Trampoline injuries occur despite adult supervision, and some studies have shown that the presence of a supervisor does not reduce injury. ${ }^{15,16)}$ However, dutiful supervision can help to enforce trampoline use rules, such as allowing only one jumper per mat and prohibiting users from performing flips. Wootton and $\mathrm{Harris}^{4)}$ warned that the presence of netted enclosures could increase the risk of injury by leading parents to believe that injuries only occur when children fall from the trampoline or make contact with the frame. This false belief is also applied to wall-towall indoor trampolines, since parents are not aware of the need for supervision for indoor trampolines. This unintended effect may be contributing to the increase in the number of injuries among the younger children. 
The single-center retrospective nature of this study imposes a few limitations, including the small number of patients involved. Our center is Ulsan's only level 1 trauma center; however, not all patients may have visited our hospitals, since some may have visited a nonED. Therefore, our cohort may not represent all trampoline-related injuries in Ulsan or Korea. In addition, variations in the level of detail of case narratives prevented us from identifying more detailed injury mechanisms and locations and from exploring the risk factors investigated in related studies, including adult supervision. Bias is also present in analysis of the risk factors, because the installation environment of each trampoline is different. Nonetheless, the study demonstrates injury characteristics and trends for trampolines in Ulsan and highlights an important emerging public health issue. Our series suggest that further injury prevention strategies are warranted. Public awareness and education are important, and legal regulations on trampoline products and installations are needed. This study is a small study, but we hope that it will facilitate safe use and help develop policy guidelines in the future.

In conclusion, during the last 10 years, trampoline-related injuries increased significantly, especially in children 6 years old. Lower extremity injuries and fractures were the most common, but injuries at various other sites were also observed. Fractures were more frequent in children $<6$ years old, especially fractures of the proximal tibia. Given the trampoline environment in Korea, prohibiting the use of trampolines for children $<6$ years old, restricting simultaneous use by multiple children, and ensuring adult supervision should be more strictly emphasized.

\section{Conflicts of interest}

No potential conflict of interest relevant to this article was reported.

\section{References}

1. Ashby K, Pointer S, Eager D, Day L. Australian trampoline injury patterns and trends. Aust N Z J Public Health 2015;39:491-4.

2. Ashby K, Eager D, D'Elia A, Day L. Influence of voluntary standards and design modifications on trampoline injury in Victoria, Australia. Inj Prev 2015;21:314-9.

3. Bhangal KK, Neen D, Dodds R. Incidence of trampoline related pediatric fractures in a large district general hospital in the United Kingdom: lessons to be learnt. Inj Prev 2006;12:133-4.

4. Wootton M, Harris D. Trampolining injuries presenting to a children's emergency department. Emerg Med J 2009;26:728-31.

5. Council on Sports Medicine and Fitness, American Academy of Pediatrics, Briskin S, LaBotz M. Trampoline safety in childhood and adolescence. Pediatrics 2012;130:774-9.

6. Trampolines at home, school, and recreational centers. American Academy of Pediatrics. Committee on Injury and Poison Prevention and Committee on Sports Medicine and Fitness. Pediatrics 1999;103(5 Pt 1):1053-6.

7. American Academy of Pediatrics. Trampolines. American Academy of Pediatrics policy statement. Itasca (IL): American Academy of Pediatrics, 1977.

8. Committee on Accident Poison Prevention. Committee on Pediatric Aspects of Physical Fitness, recreation, and Sports, American Academy of Pediatrics. Trampolines II. Pediatrics 1981;67:438-9.

9. Loder RT, Schultz W, Sabatino M. Fractures from trampolines: results from a national database, 2002 to 2011. J Pediatr Orthop 2014;34: 683-90.

10. Brown PG, Lee M. Trampoline injuries of the cervical spine. Pediatr Neurosurg 2000;32:170-5.

11. Leonard H, Joffe AR. Children presenting to a Canadian hospital with trampoline-related cervical spine injuries. Paediatr Child Health 2009; 14:84-8.

12. Choi ES, Hong JH, Sim JA. Distinct features of trampoline-related orthopedic injuries in children aged under 6 years. Injury 2018;49: 443-6.

13. Shin YW, Hong CW, Lee OJ, Park SY. The incidence of pediatric trampoline injury: statistics from a single institution. J Korean Orthop Assoc 2018;53:129-35.

14. Alexander K, Eager D, Scarrott C, Sushinsky G. Effectiveness of pads and enclosures as safety interventions on consumer trampolines. Inj Prev 2010;16:185-9.

15. McDermott C, Quinlan JF, Kelly IP. Trampoline injuries in children. J Bone Joint Surg Br 2006;88:796-8.

16. Kasmire KE, Rogers SC, Sturm JJ. Trampoline park and home trampoline injuries. Pediatrics 2016;138.

17. Mulligan CS, Adams S, Brown J. Paediatric injury from indoor trampoline centres. Inj Prev 2017;23:352-4.

18. Korea Consumer Agency. Kid cafe safety survey. Eumseong (Korea): Korea Consumer Agency, 2016.

19. Children's play facilities safety management system [Internet]. Sejong (Korea): Ministry of the Interior and Safety; [cited 2018 Jun 20]. Available from: http://www.cpf.go.kr.

20. Loder RT, Abrams S. Temporal variation in childhood injury from common recreational activities. Injury 2011;42:945-57.

21. Swischuk LE. Jumped off the trampoline: fell on knee: pain. Pediatr Emerg Care 2009;25:366-7.

22. Boyer RS, Jaffe RB, Nixon GW, Condon VR. Trampoline fracture of the proximal tibia in children. AJR Am J Roentgenol 1986;146:83-5.

23. Black GB, Amadeo R. Orthopedic injuries associated with backyard trampoline use in children. Can J Surg 2003;46:199-201.

24. Klimek PM, Juen D, Stranzinger E, Wolf R, Slongo T. Trampoline related injuries in children: risk factors and radiographic findings. World J Pediatr 2013;9:169-74.

25. Stranzinger E, Leidolt L, Eich G, Klimek PM. The anterior tilt angle of the proximal tibia epiphyseal plate: a significant radiological finding in young children with trampoline fractures. Eur J Radiol 2014;83: 1433-6.

26. Hauth E, Jaeger H, Luckey P, Beer M. MR imaging for detection of trampoline injuries in children. BMC Pediatr 2017;17:27.

27. Casserly CS, Lim RK, Prasad AN. Vertebral artery dissection causing stroke after trampoline use. Pediatr Emerg Care 2015;31:771-3.

28. Linakis JG, Mello MJ, Machan J, Amanullah S, Palmisciano LM. Emergency department visits for pediatric trampoline-related injuries: an update. Acad Emerg Med 2007;14:539-44.

29. Arora V, Kimmel LA, Yu K, Gabbe BJ, Liew SM, Kamali Moaveni A. Trampoline related injuries in adults. Injury 2016;47:192-6. 